\title{
Neutron inelastic cross section measurements on ${ }^{58,60} \mathbf{N i}$
}

\author{
Adina Olacel ${ }^{1, *}$, Catalin Borcea ${ }^{1}$, Marian Boromiza ${ }^{1}$, Philippe Dessagne ${ }^{2}$, Gregoire Henning ${ }^{2}$, Maëlle Kerveno $^{2}$, Alexan- \\ dru Negret $^{1}$, Markus Nyman ${ }^{3}$, and Arjan Plompen ${ }^{3}$ \\ ${ }^{1}$ Horia Hulubei National Institute for Physics and Nuclear Engineering, Reactorului 30, 077125 Bucharest-Măgurele \\ ${ }^{2}$ Université de Strasbourg, CNRS, IPHC UMR 7178, Strasbourg, France \\ ${ }^{3}$ European Commission, Joint Research Centre, Retieseweg 111, B - 2440 Geel, Belgium
}

\begin{abstract}
A natural nickel sample was used at the GELINA (Geel Electron LINear Accelerator) neutron source of the European Commission, Joint Research Centre, Geel to measure the neutron inelastic cross sections. The GAINS (Gamma Array for Inelastic Neutron Scattering) spectrometer was employed to detect the emitted $\gamma$ rays while a ${ }^{235} \mathrm{U}$ fission chamber monitored the neutron flux. We report the preliminary production cross sections corresponding to the first transitions in ${ }^{58,60} \mathrm{Ni}$ in comparison with previously reported data and with TALYS 1.9 calculations performed using the default input parameters.
\end{abstract}

\section{Introduction}

Very precise and reliable neutron inelastic scattering data on structural materials of nuclear reactors is very important for the design and development of these facilities. Nickel is an important component in a large number of stainless steel alloys which is a widely used material in the nuclear industry especially in the sodium cooled fast reactors and during the back end of the fuel cycle in ADS systems [1]. Natural nickel has five stable isotopes. The most abundant one is ${ }^{58} \mathrm{Ni}[68.077(6) \%]$ followed by ${ }^{60} \mathrm{Ni}$ [26.223(5)\%], ${ }^{62} \mathrm{Ni}[3.634(1) \%],{ }^{61} \mathrm{Ni}[1.139(4) \%]$ and ${ }^{64} \mathrm{Ni}[0.926(6) \%][2]$.

Our main purpose was to measure the $\gamma$-production cross sections of the most important transitions in all the stable isotopes of natural nickel and to calculate (where possible) level and total inelastic cross sections. This paper will present and discuss preliminary results of the $\gamma$ production cross sections of the first transitions in ${ }^{58} \mathrm{Ni}$ $\left(\mathrm{E}_{\gamma}=1454.28 \mathrm{keV}\right)$ and in ${ }^{60} \mathrm{Ni}\left(\mathrm{E}_{\gamma}=1332.50 \mathrm{keV}\right)$. A full publication reporting the production cross section of the other observed transitions is foreseen. The experimental results are compared with previous experimental values [3-7] taken from the EXFor (Exchange Format) data base [8] and summarized in Table 1. For the reported transitions only Voss et al. [7] measured cross sections in an extended incident energy range. Hence, we will emphasize on the comparison between our results and those of Ref. [7].

\section{Experimental setup}

The neutron inelastic scattering measurement on natural nickel was performed using the GELINA (Geel Electron

\footnotetext{
*e-mail: aolacel@tandem.nipne.ro
}

LINear Accelerator) neutron source [9-11] opperated by EC-JRC (European Commission, Joint Research Centre) Geel. This facility was especially designed for performing highly precise neutron scattering measurements using the time-of-flight technique. A linear accelerator is used to accelerate electrons $\left(\mathrm{E}_{e}=70-140 \mathrm{MeV}\right)$ with a maximum repetition rate of $800 \mathrm{~Hz}$ and a pulse duration of $1 \mathrm{~ns}$. The electrons hit a mercury-cooled depleted uranium target and produce via bremsstrahlung high energy $\gamma$ rays. This $\gamma$ rays interact with the uranium target [11]. Neutrons with energies in the range spreading from thermal up to $20 \mathrm{MeV}$ are produced via $(\gamma, x n)$ and $(\gamma, f)$ reactions inside the $\mathrm{U}$ target. GELINA is a multiuser facility with 12 flight paths distributed at various angles around the uranium target and several measurement cabins located at various distances $(10-400 \mathrm{~m})$ from the neutron source.

Our measurement was performed on flight path 3 in the measurement cabin located at $100 \mathrm{~m}$ from the neutron source and at $90^{\circ}$ with respect to the electron beam. Here, is locatted the GAINS (Gamma Array for Inelastic Neutron Scattering) spectrometer $[12,13]$ which is used to detect the $\gamma$ rays emitted following the neutron inelastic scattering on natural nickel. GAINS consists of 12 largevolume HPGe detectors having $100 \%$ relative efficiency and $2.8-\mathrm{keV}$ energy resolution at $1.33 \mathrm{MeV}$. The $12 \mathrm{de}-$ tectors are divided in groups of four, placed at 3 different angles $\left(110^{\circ}, 125^{\circ}\right.$ and $150^{\circ}$ relative to the neutron beam direction) chosen to precisely integrate the differential cross sections over the entire solid angle and using a combination of Legendre polynomials and Gaussian quadrature. The detectors are placed at backward angles with respect to the sample in order to reduce the impact of the $\gamma$ rays emitted from the photon flash. The neutron flux was monitored using a ${ }^{235} \mathrm{U}$ fission chamber (FC) centered on the beam at about $211.5 \mathrm{~cm}$ upstream the sample and it contains 8 uranium layers of $70 \mathrm{~mm}$ diameter 
Table 1. Summary of previous experiments on neutron inelastic scattering on ${ }^{58,60} \mathrm{Ni}$ available in the ExFor data base [8]. The table also displays the year when the data was reported, the covered neutron energy range, the detectors used in the experiment and the EXFOR entry.

\begin{tabular}{lrrccc}
\hline Reference & Year & Sample & $\begin{array}{c}\mathrm{E}_{n} \text { range } \\
(\mathrm{MeV})\end{array}$ & Detectors & EXFOR entry \\
\hline D.A. Bazavov et al. [3] & 1983 & ${ }^{58} \mathrm{Ni}$ & $1.9-3.0$ & $\mathrm{GeLi}$ & 40736 \\
D.L. Broder et al. [4] & 1964 & ${ }^{58,60} \mathrm{Ni}$ & $1.3-4.1$ & $\mathrm{NaI}$ & 40139 \\
E.S. Konobeevskii et al. [5] & 1972 & ${ }^{58,60} \mathrm{Ni}$ & $1.3-1.6$ & $\mathrm{GeLi}$ & 40096 \\
G. Tessler et al. [6] & 1975 & ${ }^{60} \mathrm{Ni}$ & $3.4-5.4$ & $\mathrm{GeLi}$ & 10439 \\
F. Voss et al. [7] & 1975 & ${ }^{58,60} \mathrm{Ni}$ & $1.3-13.5$ & $\mathrm{GeLi}$ & 20744 \\
\hline
\end{tabular}

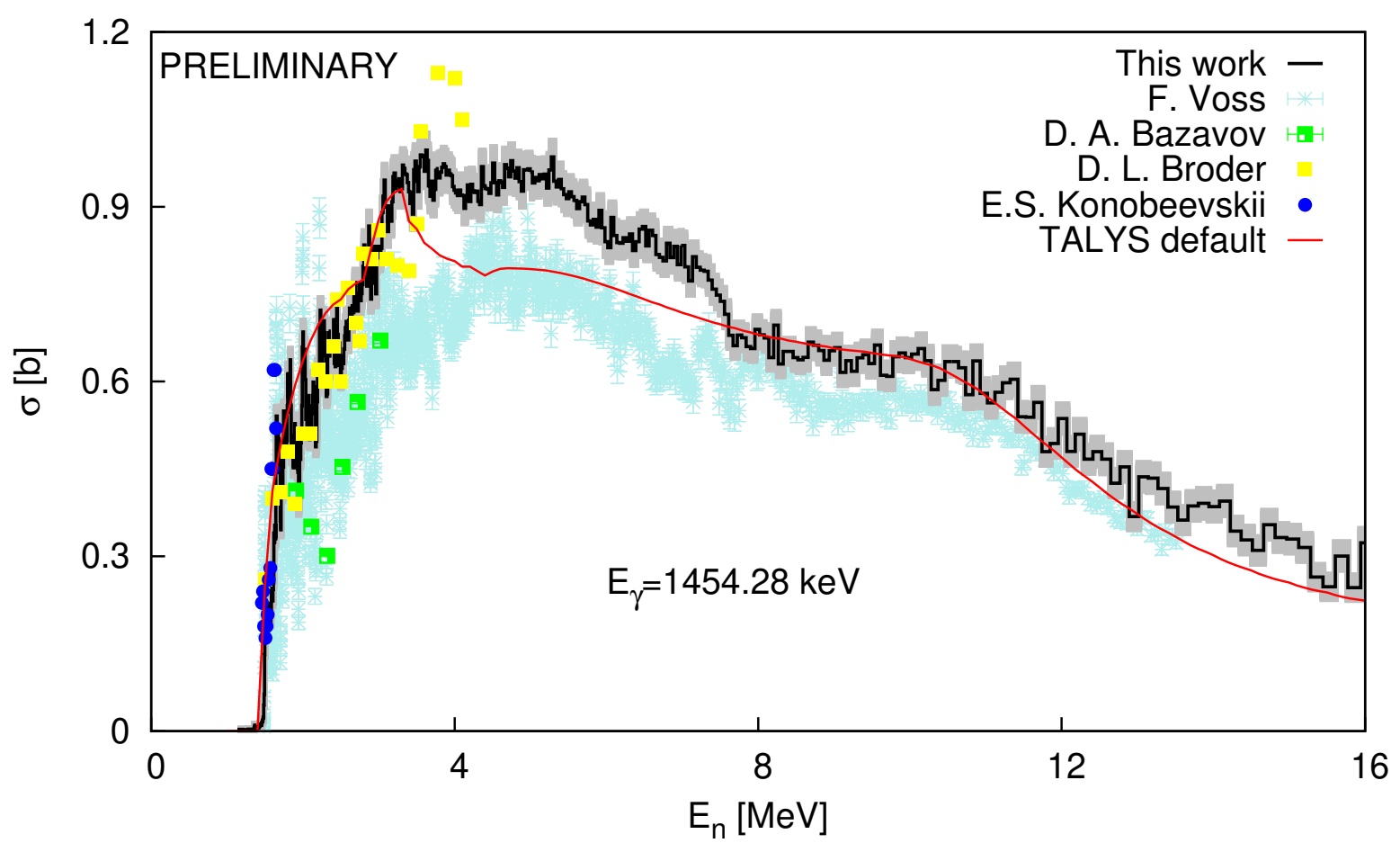

Figure 1. (Color online) The $\gamma$-production cross section of the main transition in ${ }^{58} \mathrm{Ni}$. Our experimental results are compared with previous experimental values and theoretical calculations performed using TALYs 1.9 code.

on five Al foils of $20-\mu \mathrm{m}$ thickness supported by a $84-$ $\mathrm{mm}$ diameter ring [14]. The working principle of the FC is extensively described in Refs. [14-16]. GAINS uses Acqiris DC440 digitizers running at $420 \times 10^{6}$ samples/s (2.38 ns/sample), with 12-bit amplitude resolution (4096 channels full range) while the acquisition of the $\mathrm{FC}$ is analogue.

\subsection{Experimental particularities}

The natural nickel sample with a diameter of 8.000(1) $\mathrm{cm}$ and a $0.3-\mathrm{cm}$ thickness was irradiated for $\approx 300 \mathrm{~h}$. The areal density of the sample $\left[2.661(21) \mathrm{g} / \mathrm{cm}^{2}\right]$ was used together with the isotopic abundances to determine the effective areal densities corresponding to ${ }^{58} \mathrm{Ni}$ $\left[1.812(15) \mathrm{g} / \mathrm{cm}^{2}\right]$ and to ${ }^{60} \mathrm{Ni}\left[0.698(5) \mathrm{g} / \mathrm{cm}^{2}\right]$. The FC had a calculated efficiency of $85.5(17) \%$ uncertainty [17]. For the calibration measurements we used a ${ }^{152} \mathrm{Eu}$ point- like source with an activity of $18.6(6) \mathrm{kBq}$. For the efficiency calibration we employed a procedure which combines experimental measurements (using a ${ }^{152} \mathrm{Eu}$ pointlike source) and Monte Carlo simulations (to take into consideration the geometric and the attenuation effects inside the extended sample). Monte Carlo simulations were also used in order to account for the multiple scattering of the neutrons in the target and in the surrounding materials.

\section{Results and discussion}

Figure 1 displays the preliminary $1454.28-\mathrm{keV} \quad \gamma$ production cross section from ${ }^{58} \mathrm{Ni}$. Our experimental results are compared with other experimental values reported in Refs. [3-5, 7] and with theoretical calculations performed with TALYs 1.9 code [18] using default input parameters. The values predicted by TALYs 1.9 describe very well our experimental cross section for most of the energy 


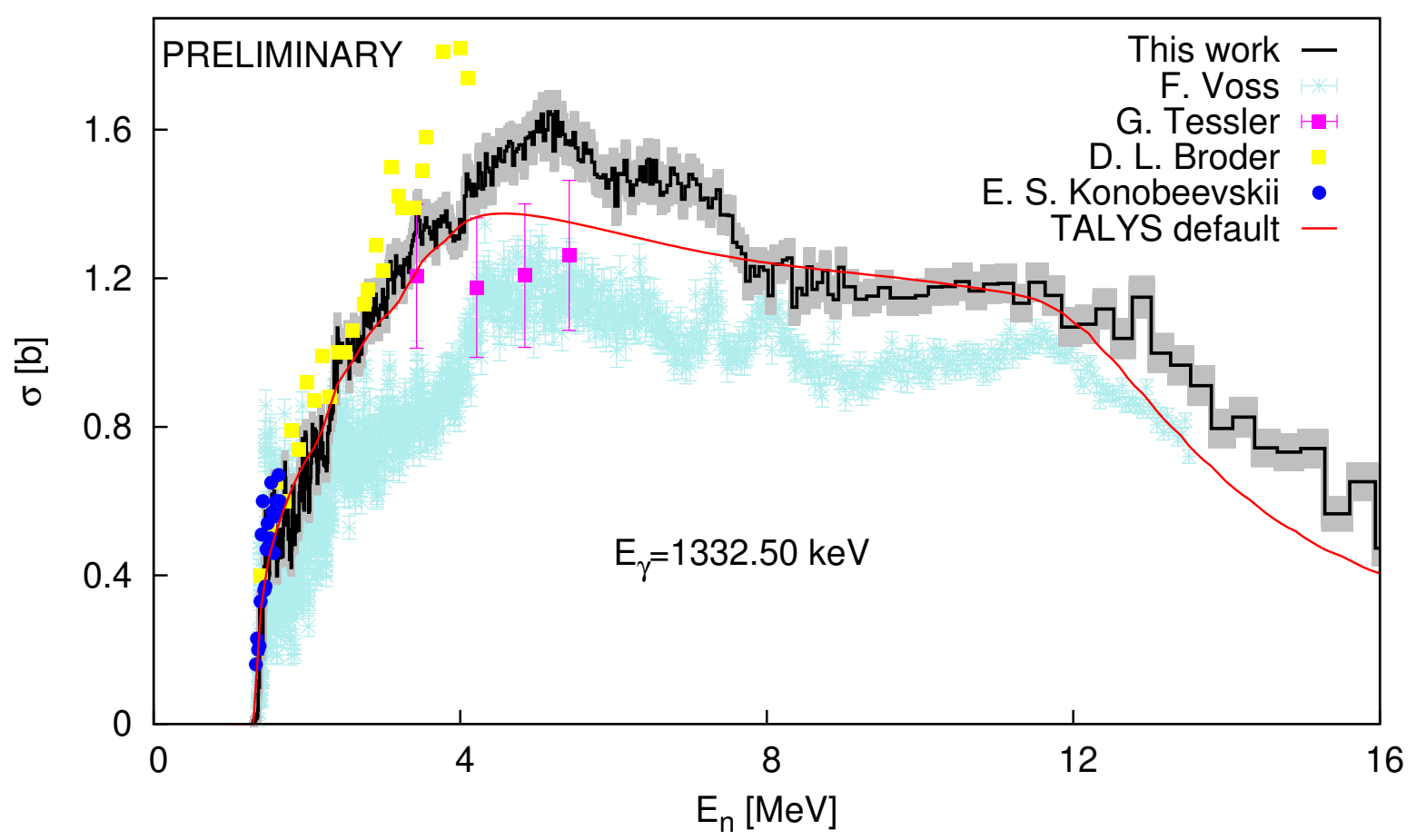

Figure 2. (Color online) The $\gamma$-production cross section of the main transition in ${ }^{60} \mathrm{Ni}$. Our experimental results are compared with previous experimental values and theoretical calculations performed using TALYs 1.9 code.

range except a region between 3.0-7.5 $\mathrm{MeV}$. The present data for this transition yields a maximum value around $1 \mathrm{~b}$ and the cross section stays significant even at the high neutron energies. The data sets reported in Refs. [4, 5] are in a good agreement with our experimental values while the ones measured by D.A. Bazavov et al. [3] and F. Voss et $a l$. [7] underestimate the present results even though the shape is similar.

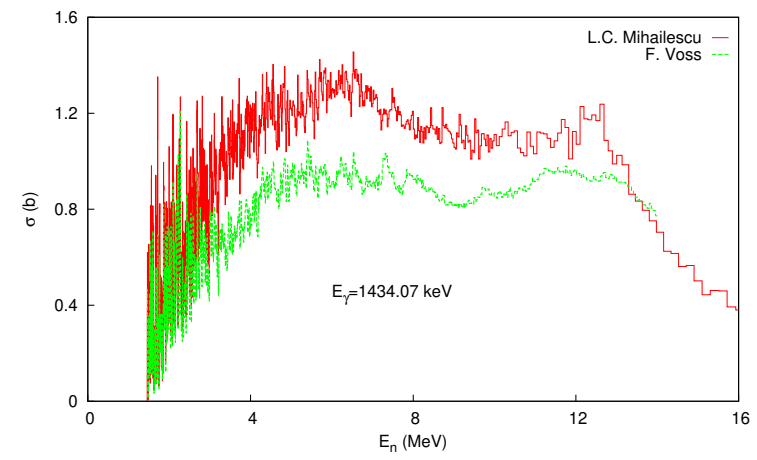

Figure 3. (Color online) The production cross section of the main transition in ${ }^{52} \mathrm{Cr}$. The data are taken from the EXFOR database.

The $\gamma$-production cross section of the main transition in ${ }^{60} \mathrm{Ni}$ is shown in figure 2 (preliminary results). Similar with the case of the $1454.28-\mathrm{keV}$ transition, the results of F. Voss et al. underestimate our data while the ones from Refs. [4, 5] overlap with our values especially bellow
3.5 MeV. The theoretical prediction underestimates the experimental values in the same energy range as for the main transition in ${ }^{58} \mathrm{Ni}$. The neutron inelastic cross section for this transition displays almost double values as compared with the $1454.28-\mathrm{keV} \gamma$ ray in ${ }^{58} \mathrm{Ni}$.

Considering that the present and the F. Voss et al. data are the only results in the entire incident energy range relevant for the inelastic channel, this motivated an additional investigation. We notice $\mathrm{a} \approx 20 \%$ difference between the two experiments. Interestingly, the same difference was also observed for two other data sets that reported inelastic cross sections on ${ }^{52} \mathrm{Cr}[7,19]$ (see figure 3). This nucleus was measured by Voss et al. using the same setup as for nickel [7] while Mihailescu et al. used the same facility (GELINA neutron source), a similar experimental setup and a $200 \mathrm{~m}$ flight path as compared with the present work. The data of Mihailesc et al., taken from the EXFOR database, reveal a similar difference of around $20 \%$ when compared with the data from Ref. [7] for ${ }^{52} \mathrm{Cr}$. The presence of this discrepancy in all these measurements suggests a systematic error in normalization of the data reported by F. Voss et al. [7].

Our results are compared also with theoretical calculations performed with the TALYS 1.9 code using the default input parameters. These use the semi-empirical model and parameters obtained from global optimizations. The level densities are calculated using the approach of Gilbert and Cameron [20] with the constant temperature model at lower energies and the back shifted Fermi gas model with one energy dependent level density parameter which ac- 
counts for the damped shell effect at higher energies. The generalized Lorentzian form by Kopecky and Uhl [21] describes the $\gamma$-ray strength functions for the E1 transitions while the Brink-Axel option [22, 23] is used to describe the other multipolarities. The nuclear structure and the decay table used in the modeling of de-excitation of the nuclei is derived from The Reference Input Parameter Library [24].

\section{Conclusions}

Using the GELINA neutron source and the GAINS spectrometer we detected several transitions coming from the inelastic scattering of neutrons by a natural nickel sample. Here, we presented only the $\gamma$-production cross section of the main transitions emitted from the two most abundant isotopes of natural $\mathrm{Ni}\left({ }^{58,60} \mathrm{Ni}\right)$. These results were compared with the default TALYs calculations and with previous available results. A special discussion was done on the comparison with the data reported in Ref. [7], the only other data covering almost the same extended energy range as our values. The comparison reveals a normalization issue in the data reported by Voss et al..

This work was supported by the European Commission within the Seventh Framework Programme through Fission2013-CHANDA (project no. 605203) and by the Ministry of Research and Innovation of Romania, CNCS-UEFISCDI, through project no. PN-III-P4-ID-PCE-2016-0025 within PNCDI III. The authors acknowledge the support team of the GELINA facility for providing stable conditions for the experiment.

\section{References}

[1] Baldev Raj, U. Kamachi Mudali, M. Vijayalakshmi, M.D. Mathew, A.K. Bhaduri, P. Chellapandi, S. Venugopal, C.S. Sundar, B.P.C. Rao, B. Venkataraman, Advanced Materials Research Vol. 794, 3-25 (2013).

[2] J. Meija, T.B. Coplen, M. Berglung, W.A. Brand, P. De Bièvre, M. Gróning, N.E. Holden, J. Irrgeher, R.D. Loss, T. Walczyk, and T. Prohaska, Pure Appl. Chem. 88(3), 293-306 (2016).

[3] D.A. Bazavov, I.E. Kashuba, V.P. Prikhod'ko, A.A. Golubova, S.V. Stoljarov, Ukrainskii Fizichnii Zhurnal, Vol.28, Issue.12, p.1791 (1983).

[4] D.L. Broder, V.E. Kolesov, A.I. Lashuk, I.P. Sadokhin, A.G. Dovbenko, Atomnaya Energiya, Vol.16, Issue.2, p.103 (1964).

[5] E.S. Konobeevskii, R.M. Musaelyan, V.I. Popov, I.V. Surkova, Bull.Russian Academy of Sciences - Physics, Vol.35, p.2127 (1972)

[6] G. Tessler, and S.Glickstein, Conf.on Nucl.CrossSect.and Techn.,Washington 1975, Vol.2, p.934 (1975).

[7] F. Voss, S. Cierjacks, D. Erbe, G. Scmalz, Conf.on Nucl.Cross-Sect.and Techn.,Washington 1975, p.916 (1975).
[8] N. Otuka et al., Nucl. Data Sheets 120, 272 (2014).

[9] A. Bensussan, J. M. Salome, Nucl. Instrum. Methods 155, 11 (1978).

[10] M. Flaška, Ph.D. thesis, Slovak University of Technology in Bratislava, Published by IOS Press under the imprint Delft University Press, 2006.

[11] D. Ene, C. Borcea, S. Kopecky, W. Mondelaers, A. Negret, A. J. M. Plompen, Nucl. Instrum. Methods Phys. Res. A, Vol. 618, 54 (2010).

[12] D. Deleanu, C. Borcea, Ph. Dessagne, M. Kerveno, A. Negret, A. J. M. Plompen, and J.C. Thiry, Nucl. Instrum. Methods Phys. Res. 624, 130 (2010).

[13] A. Negret, C. Borcea, Ph. Dessagne, M. Kerveno, N. Nankov, A. Olacel, A. J. M. Plompen, and C. Rouki, Nucl. Data Sheets 119, 179 (2014).

[14] L. C. Mihailescu, L. Olah, C. Borcea, and A. J. M. PLompen, Nucl. Instrum. Methods Phys. Res. A 531, 375 (2004).

[15] C. Rouki, P. Archier, C. Borcea, C. De Saint Jean, J. C. Drohé, S. Kopecky, A. Moens, N. Nankov, A. Negret, G. Noguère, A. J. M. Plompen, and M. Stanoiu, Nucl. Instrum. Phys. Res. A 672, 82 (2012).

[16] Adina Olacel, Catalin Borcea, Marian Boromiza, Philippe Dessagne, Gregoire Henning, Maëlle Kerveno, Luiz Leal, Alexandru Negret, Markus Nyman, and Arjan Plompen in Proceedings of International Conference on Nuclear Data for Science and Technology, Beijing, China, 2019.

[17] A. Plompen, N. Nankov, C. Rouki, M. Stanoiu, C. Borcea, D. Deleanu, A. Negret, Ph. Dessagne, M. Kerveno, G. Rudolf, J.C. Thiry, M. Mosconi, R. Nolte, J. Korean Phys. Soc. 59, 1581 (2011).

[18] A. J. Koning, S. Hilaire and M. C. Duijvestijn, International Conference on Nuclear Data for Science and Technology, April 22-27, 2007, Nice, France, editors O. Bersillon, F. Gunsing, E. Bauge, R. Jacqmin, and S. Leray, EDP Sciences, p. 211-214 (2007).

[19] L.C. Mihailescu, C. Borcea, A.J. Koning, A.J.M. Plompen, Nuclear Physics A 786, 1-3 (2007).

[20] A. Gilbert and A. G. W Cameron, Can. J. Phys. 43, 1446 (1965).

[21] J. Kopecky and M. Uhl, Phys. Rev. C 41, 1941 (1990).

[22] D. M. Brink, Ph.D. thesis, Oxford University, 1955.

[23] P. Axel, Phys. Rev. 126, 671 (1962).

[24] R. Capote, M. Herman, P. Obložinský, P. G. Young, S. Goriely, T. Belgya, A. V. Ignatyuk, A. J. Koning, S. Hilaire, V. A. Plujko, M. Avrigeanu, O. Bersillon, M. B. Chadwick, T. Fukahori, Zhigang Ge, Yinlu Han, S. Kailas, J. Kopecky, V. M. Maslov, G. Reffo, M. Sin, E. Sh. Soukhovitskii, P. Talou, Nucl. Data Sheets 110, 3107 (2009). 